\title{
STABILISATION DE LA RÉGULATION DES SOURCES DE REVENUS DES COLLECTIVITÉS TERRITORIALES EN POLOGNE À LA LUMIĖRE DE LA CONSTITUTION DE LA RÉPUBLIQUE DE POLOGNE ET LA CHARTE EUROPÉENNE DE L'AUTONOMIE LOCALE
}

\section{Introduction}

La notion de la stabilisation est utilisée dans le présent travail au sens d'un ordre déterminé et de l'état de l'équilibre, du maintien des solutions adoptées pendant un temps prolongé ${ }^{1}$. En Pologne, dès le moment de la réactivation de l'administration locale décentralisée, c'est-à-dire à partir de 1990, continue le processus de perfectionnement des principes et méthodes du financement des devoirs des collectivités locales. Jusqu'en 1994, ce processus était en danger des changements temporels parce qu'il n'y avait pas de standards formels en vigueur qui auraient déterminé des modèles précis des revenus des collectivités locales, puisque la Constitution de 1952 ne comprenait aucune décision en cette matière. Seulement dans l'article 73 alinéa 2 de la loi constitutionnelle de $1992^{2}$, a été introduite la règle selon laquelle les sources de revenus des collectivités locales dans le domaine des devoirs publics sont garanties par la loi. Dans l'article mentionné on peut percevoir les premières directives indiquant le besoin de créer des solutions stables dans le domaine du système des revenus des collectivités locales.

L'achèvement au 1 mars 1994 de la procédure de ratification ${ }^{3}$ de la Charte européenne de l'autonomie locale par la Pologne était une étape importante

Słownik języka polskiego (Dictionnaire de la Langue Polonaise) vol.5, (dir. M. Bańko), Wydawnictwo Naukowe PWN. Varsovie 2007, p.165.

2 Loi Constitutionnelle du 17 octobre 1992 sur les relations réciproques entre le pouvoir législatif et exécutif de la République de Pologne et sur l'autonomie locale (Dz. U. Nr 84, poz. 426 ze zm.), en vigueur jusqu'au 17 octobre 1997.

3 Déclaration de Gouvernement du 14 juillet 1994 dans la matière de la ratification de la Charte européenne de l'autonomie locale, faite à Strasbourg le 15 octobre 1985 (Dz. U. Nr 124, poz. 608). 
de ces changements. Dans l'article 9 de la C.E.A.L. ${ }^{4}$ on a formulé des principes fondamentaux du système financier des collectivités locales. On peut y voir aussi le principe de stabilisation des bases de l'économie financière des collectivités locales, surtout par rapport aux décisions de l'article 9 de la C.E.A.L., où l'on a fait rappel à la loi comme garantie d'adapter le niveau des ressources financières des collectivités locales au domaine de leurs compétences, d'exercer l'autorité fiscale, d'avoir l'accès au marché des capitaux national.

Pourtant, c'est seulement le vote de la Constitution de la RP en $1997^{5}$ qui a consolidé le principe mentionné, qui devrait actuellement être considéré comme standard suprême au moment de créer les modèles des revenues des collectivités locales en Pologne. Il est aussi important que le principe de stabilisation a été visiblement associé à d'autres principes constitutionnels importants qui concernent directement l'économie financière des collectivités locales, c'est-à-dire au principe de la compatibilité des ressources financières et des devoirs (dans le cadre statique et dynamique), de la priorité des revenus propres sur les revenus subventionnels (subventions générales et dotations spécifiques du budget national) et de l'autorité fiscale, déterminée par les lois. La détermination de tels standards directement dans les actes constitutionnels satisfait aux postulats avancés depuis plusieurs années par les représentants de la doctrine, proposant que le système des finances locales respecte les conditions de clarté, de stabilité et de compatibilité dans le domaine du financement des objectifs confiés à la collectivité locale ${ }^{6}$. La nécessité de régir par les lois la création des sources de revenus est fortement accentuée dans la doctrine ${ }^{7}$.

\section{La loi comme "garantie » de la stabilisation des ressources financières des collectivités locales}

Dans les conditions polonaises, à cause de l'aisance d'initier des régulations législatives et du prétendu idéalisme administratif (conviction que l'établissement ou le changement des standards législatifs peut entraîner des changements souhaités dans la vie sociale) il faut rappeler sans cesse que les moyens législatifs ne sont qu'un moyen parmi d'autres d'influencer la vie sociale ${ }^{8}$. Formellement, on se référait à la loi comme à un moyen législatif qui régule les bases de l'économie financière

\footnotetext{
4 Charte européenne de l'autonomie locale, faite à Strasbourg le 15 octobre 1985 (Dz. U. z 1994 r. Nr 124, poz. 607).

$5 \quad$ Constitution de la République de Pologne du 2 avril 1997 (Dz. U. Nr 78, poz. 483 ze zm.).

6 Cz. Rudzka - Lorentz, H. Sochacka - Krysiak, Źródła finansowania lokalnych zadań publicznych a problem samodzielności jednostek samorządu terytorialnego (w:) Gospodarka jednostek samorządu terytorialnego w warunkach decentralizacji zarządzania sektorem publicznym, sous la dir. de H. Sochacka-Krysiak, Varsovie 2008, p. 139.

7 W. Miemiec, Prawne gwarancje samodzielności finansowej gminy w zakresie dochodów publicznoprawnych, Wrocław 2005, p. 90.

8 S. Wronkowska, M. Zieliński, Komentarz do zasad techniki prawodawczej, Varsovie 2004, p. 23.
} 
des collectivités locales mais, dans la période qui précède l'entrée en vigueur de la Constitution de la RP de 1997, on n'apercevait souvent pas le besoin de stabilisation des solutions appliquées dans ce domaine.

Dans les années 1990-1998, il y avaient deux lois régulant les principes de l'économie financière des communes : la loi du 14 décembre 1990 sur les revenues des communes et les principes de leur subventionnement dans les années 1991$1993^{9}$, et la loi du 10 décembre 1993 sur le financement des communes ${ }^{10}$, entrée en vigueur le $1^{\text {er }}$ janvier 1994, mais qui a perdu sa force obligatoire à la fin de 1998. Un trait caractéristique pour cette période était la temporalité des solutions législatives, qui est malheureusement devenue un standard et une méthode d'établir des sources des revenues des collectivités dans le années suivantes ${ }^{11}$. La première des lois mentionnées était renouvelée trois fois, et la deuxième treize fois (dans ce cas, on a introduit en plus une période de transition de deux ans).

L'acte suivant, aussi temporaire par principe, aurait dû être en vigueur pendant deux ans seulement, mais cette période a été trois fois prolongée ${ }^{12}$. Cet acte a été amendé huit fois. A partir du $1^{\text {er }}$ janvier 2004, il y a la loi du 13 novembre 2003 sur les revenus des collectivités locales ${ }^{13}$ qui est en vigueur. Cependant, il faut souligner qu'elle introduisait des solutions temporaires pour les années 2004-2005, et qu'elle a été en plus amendée déjà six fois.

Dans les années 1990-2008, il y avait quatre lois en vigueur, régulant le système des revenues des collectivités locales, qui étaient amendées au total trente fois. Cela ne favorisait certainement pas la stabilisation des solutions relatives aux sources des revenues des collectivités locales mais, au contraire, a renforcé l'opinion à propos de l'instabilité de ces solutions, de la création des régulations temporaires et passagères, visant des objectifs ad hoc. L'admission du juste principe selon lequel la loi - comme moyen législatif - devrait consolider les solutions relatives au système des revenus des collectivités locales pour une période prolongée, n'a pas conduit à la réalisation de ces objectifs.

Le problème est sérieux et ne devrait pas être amené au seul phénomène d'initiative législative relativement simple et de dévalorisation de la loi en tant qu'un moyen législatif appliqué en Pologne. Il faut en chercher des raisons dans le manque d'une conception de l'autorité territoriale en Pologne cohérente. Des prétendues reformes de l'autorité locale que l'on voit de temps en temps surgir ne devrait pas se concentrer sur le nombre des collectivités, comme par exemple la discussion de

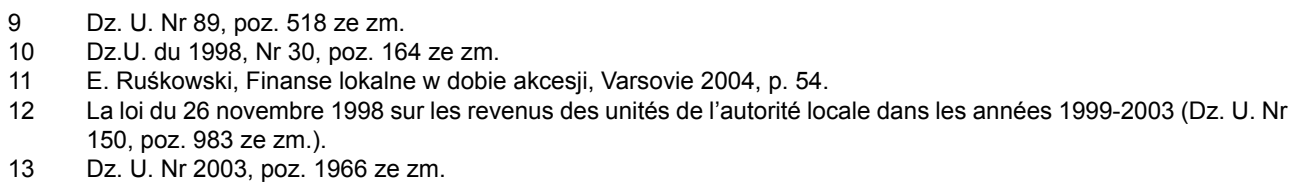


Stabilisation de la régulation des sources de revenus des collectivités territoriales...

1998 sur le nombre de districts et de voïvodies ou, depuis 2007 sur le nombre de métropoles.

C'est le problème du domaine de la décentralisation dans la réalisation des objectifs publics, de la détermination des frais de leurs réalisation et des méthodes visant à satisfaire d'une manière optimale les besoins des communautés locales qui est d'une importance fondamentale. Il faut en plus prendre en considération les possibilités de financement de ces objectifs avec les moyens publics. On ne peut donc pas oublier, dans la discussion sur le modèle des revenus des collectivités locales, la situation de tout le secteur des finances publiques. Le sous-secteur des finances locales est bien un des éléments du secteur des finances publiques, qui est en plus composé du sous-secteur gouvernemental et du sous-secteur des assurances sociales. Tous les trois sont dépendants les uns des autres. Il faut donc retenir que même les moindres changements effectués dans l'un des sous-secteurs peuvent influencer non seulement ce sous-secteur, mais aussi deux autres. De tels changements peuvent donner des résultats pareils a ceux que provoque une pierre jetée dans l'eau et faisant apparaître des cercles à la surface.

\section{Changements temporaires ou "grande" réforme des finances locales?}

Les expériences vécues prouvent qu'on a profité plus souvent de la méthode des changements temporaires, en adaptant le système des revenus des collectivités locales aux phénomènes survenus. Il est caractéristique que seulement en 2003, en présentant les principes du projet de loi sur les revenus des collectivités locales, on a constaté que c'étaient des solutions à caractère définitif. On estimait à l'époque que le vote d'une loi devrait être une étape importante sur la voie à une meilleure décentralisation et à la consolidation de l'autonomie locale en Pologne. Elle devrait aussi constituer un élément important du programme de la réparation des finances publiques ${ }^{14}$. Les projets de loi sur les revenus des collectivités locales antérieurs non seulement ne comprenaient de telles précisions, mais on y soulignait en plus la temporalité des solutions proposées.

Il est incontestable que le système des revenus des collectivités locales, déterminé par la loi du 13 novembre 2003 n'est pas parfait et n'assure pas la réalisation de l'un des principes fondamentaux de la prétendue économie financière autonome ${ }^{15}$. La stabilisation de l'économie financière demande d'entreprendre plusieurs actions,

\footnotetext{
14 Justification du projet de la loi sur les revenus des collectivités locales - document numéro 1732 de la Chambre des Députés de la RP de la IV législature.

15 A comparer l'art. 51al. 1 de la loi du 8 mars 1990 sur l'autonomie communale (Dz. U. z 2001 r. Nr 142, poz. 1591 ze zm.), l'art 51 de la loi du 5 juin1998 sur l'autonomie du district (Dz. U. z 2001 r. Nr 142, poz. 1592 ze zm.), et l'art. 6 de la loi du 5 juin 1998 sur l'autonomie de la voïvodie (Dz. U. z 2001 r. Nr 142, poz. 1590 ze zm.).
} 
dont l'augmentation de l'importance des sources des revenus propres dans le financement des objectifs des districts et des voïvodies, l'élimination des restes des mécanismes d'estimation dans l'attribution des subventions générales, la réduction du nombre des dotations spécifiques du budget de l'Etat, le renforcement du rôle de la représentation des collectivités locales dans le processus de subventionner cellesci (par exemple remplacer la présente opinion par l'exigence d'obtenir une opinion positive de la représentation par un ministre compétent), l'introduction du mécanisme de remboursement des revenus perdus à la suite des changements défavorables pour la collectivité locale dans les actes régulant les principes d'exécution des revenus propres des collectivités locales, la régulation conséquente des principes de financement des objectifs des collectivités locales dans les lois et non dans les actes exécutifs.

La préparation d'un nouvel acte sur les revenus des collectivités locales n'est conseillé que s'il prend en compte tous les standards résultant des dispositions de la Constitution de la RP et la C.E.A.L. Cela devrait être précédé d'une analyse minutieuse des effets de la législation en vigueur jusqu'à présent, et surtout d'une analyse de la jurisprudence du Tribunal Constitutionnel, des tribunaux administratifs et des chambres de compte régionales. Il faut définir précisément les objectifs visés par la nouvelle loi sur les revenus des collectivités locales.

Tenant compte des décisions du $\S 2$ des principes de la technique législative ${ }^{16}$, selon lesquels un acte devrait réguler d'une manière exhaustive un domaine donné, sans laisser de côté des fragment essentiels de ce domaine, il faut réfléchir sur l'intégration de plusieurs règlements qui se trouvent actuellement sous la loi sur les revenus des collectivités locales. Cela peut concerner les principes de transmission de la part compensatrice de la subvention générale aux communes, à titre de la perte d'une partie des recettes fiscales sur les biens immeubles ${ }^{17}$. En plus, il faut formuler correctement dans ce nouvel acte les dispositions qui renvoient aux autres lois, régulant les principes d'accorder les dotations pour les collectivités locales (actuellement, les principes d'accorder les dotations ont été régulés dans plus d'une douzaine d'actes, par exemple dans le domaine de l'organisation et de l'exécution des objectifs dans le domaine de la culture, de la conservation des monuments, du rapatriement, et aussi les dotation pour les communes thermales).

A l'occasion de la préparation d'une nouvelle loi sur les revenus des collectivités locales, il faut aussi éliminer de nombreux règlements incohérents concernant l'économie financière des collectivités locales, présents dans les lois sur l'autorité communale et de district, et dans la loi sur l'autorité de voïvodie. Les actes mentionnés

\footnotetext{
16 Arrêté du Premier ministre du 20 juin 2002 relatif aux „Principes de la technique législative” (Dz. U. Nr 100, poz. 908).

17 Ces questions sont actuellement réglées par la loi du 2 octobre 2003 sur le changement de la loi sur les zones économiques spéciales et certaines autres lois (Dz. U. Nr 188, poz. $1840 \mathrm{ze} z \mathrm{~m}$.).
} 
Stabilisation de la régulation des sources de revenus des collectivités territoriales...

utilisent des notions et la structure des ressources des collectivités locales qui ne correspondent pas aux standards admis dans la loi sur les revenus : les revenus stables et extraordinaires y sont par exemple classés autrement, on utilise des notions qui ne sont pas compatibles à celles de la loi sur les revenus (par exemple subvention compensatrice au lieu de partie compensatrice de la subvention générale). Il faut ajouter en outre que le classement des sources des revenus des collectivités locales, appliqué dans les lois sur l'autorité communale et de district, ainsi que dans la loi sur l'autorité de voïvodie ne tient pas compte des critères de la division de ces sources, appliqués dans l'article 167 de la Constitution de la RP, conformément auxquels les revenus propres, les subventions générales et les dotations spécifiques du budget de l'Etat ont un caractère stable. Dans les lois régulant régime, objectifs et bases de l'économie financière de l'autorité locale, les dotations spécifiques sont traitées comme sources des revenus de caractère extraordinaire.

Tenant compte des dispositions du $\S 4$ alinéa 1 des principes de la technique législative, selon lesquelles une loi ne peut pas répéter les dispositions des autres lois, il faut supprimer de nombreuses répétitions se référant aux étapes de la procédure budgétaire. Aussi bien dans les lois sur l'autorité communale et de district, que dans la loi sur l'autorité de voïvodie et la loi sur les finances publiques ${ }^{18}$ se trouvent des dispositions pareils ou même identiques, régulant la préparation du projet de budget des collectivités locales et le vote de la loi budgétaire. Les règlements de procédure devraient être inscrits dans la même loi, par exemple loi sur les finances publiques.

En préparant le projet d'une nouvelle loi sur les revenus des collectivités locales, il faut normaliser exhaustivement les principes de l'économie financière, surtout dans le domaine des revenus des fédérations intercommunales et fédérations de districts. Le maintien des solutions actuelles, qui consistent à imposer l'application des dispositions concernant l'économie financière des communautés et districts, peut être considéré comme un exemple d'un attachement exagéré et irrationnel du législateur aux régulations provisoires. La spécificité du fonctionnement des fédérations, en tant qu'une forme la plus stricte et la plus avancée de coopération des collectivités locales ne fait pas douter, que les fédérations sont des sujets de loi distinctes des collectivités qui les forment ${ }^{19}$. Cela devrait trouver un reflet adéquat dans des régulations propres et intégrales des principes de l'économie financière des fédérations dont l'importance ne cesse d'augmenter.

La préparation d'une nouvelle loi cohérente sur les revenus des collectivités locales, de la loi sur l'économie financière de celles-ci et de leurs fédérations, normalisant non seulement les sources de revenus mais aussi les principes de

18 Loi du 30 juin 2005 sur les finances publiques (Dz. U. Nr 249, poz. 2104 ze zm.).

19 M. Ofiarska, Formy publicznoprawne współdziałania jednostek samorządu terytorialnego, Varsovie 2008, p. 441. 
financement des objectifs propres et délégués, la création et l'application des procédures compensatrices et équilibrantes, la surveillance et le contrôle dans le domaine financier et les règles de la participation au marché des capitaux, exige de longues préparations, des consultations sociales et des analyses économiques et financières approfondies. C'est pourquoi on peut s'attendre dans les années à venir à ce que les réformes et les changements partiels continuent, forcés souvent par les verdicts du Tribunal Constitutionnel et la pression des milieux locaux, mais aussi par le lobbying des collectivités locales au Parlement. C'est n'est pourtant qu'un substitut des réformes. La réforme des finances locales en Pologne devrait être associée à une réforme structurale de tout le secteur des finances publiques ${ }^{20}$.

\section{Tendances principales des changements temporaires dans les années 2006-2008}

Apres l'achèvement de la prétendue période transitoire, qui a duré deux ans à partir du moment de l'entrée en vigueur de la loi sur les revenus des collectivités locales, on peut apercevoir des actions qui résultent en l'affaiblissement progressif $\mathrm{du}$ potentiel des revenu des collectivités locales, surtout des communes. A partir du

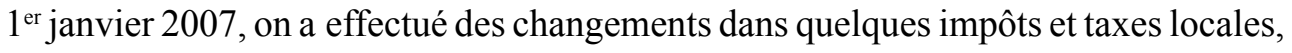
sources de revenus propres des communes, qui peuvent à long terme provoquer une diminution considérable des revenus budgétaires.

En amendant ${ }^{21}$ la loi relative à 1 ' impôt sur l'héritage et les donations ${ }^{22}$, on a introduit une exemption de l' impôt essentiel, qui consiste à éliminer la charge de l'impôt des personnes qui acquièrent gratuitement les biens des personnes les plus proches. Guidé par le besoin de la protection particulière de la situation matérielle de la famille, et par le fait que la transmission gratuite de la propriété a en principe lieu entre les personnes les plus proches, le législateur a adopté les solutions qui mènent a une exemption totale de l' impôt l'acquisition gratuite de la propriété des personnes les plus proches. On a admis que les liens personnels et familiaux particuliers entre les parents en ligne directe et entre les époux justifient l'exemption totale de l' impôt en cas de l'acquisition des choses ou des droits patrimoniaux par l'époux, les descendants, ascendants, beau-fils et belle-fille, frères et soeurs, beaupère et belle-mère. Cette exemption dépend des conditions formelles imposées à l'acquéreur (déclaration dans le délai prévu de l'acquisition à la trésorerie compétente, acquisition de la donation en argent sur le compte bancaire ou par mandat postal).

20 E. Ruśkowski, J.M. Salachna, Finanse lokalne po akcesji, Varsovie 2007, p. 267.

21 Loi du 16 novembre 2006 sur le changement de la loi sur l'impôt sur les héritages et donations et de la loi sur l'impôt sur les actes de droit civil (Dz. U. Nr 222, poz. 1629).

22 Loi du 28 juillet 1983 sur l'impôt sur les héritages et donations (Dz. U. z 2004 r. Nr 142, poz. 1514 ze. zm.). 
Stabilisation de la régulation des sources de revenus des collectivités territoriales...

On a introduit aussi d'autres changements menant à élargir les limites des dégrèvements et exemptions de l'impôt sur l'héritage et les donations (par exemple dans l'article 4 alinéa 1 numéro 5 de la loi, on a élargi le catalogue des objectifs qui conditionnent l'exemption des donations acquises par les personnes du premier groupe d'impôt, en y ajoutant le remboursement du crédit de logement hypothécaire avec les intérêts; on a ajouté une nouvelle disposition à l'article 4 alinéa 1 numéro 5a de la loi, selon lequel est exempte de l'impôt l'acquisition par voie héréditaire des droits à l'investissement résidentiel dans une société coopérative par les personnes appartenant au premier ou deuxième groupe d'impôts, qui satisfont aux conditions donnant le droit à une remise résidentielle; on a introduit en plus des conditions plus favorables, qui permettent de garder le droit à la remise résidentielle décrite dans l'art. 16 de la loi, en prolongeant de six mois à deux ans la période dans laquelle on devrait acquérir un nouveau bâtiment ou local avec les moyens acquis de la vente du local ou bâtiment précédent).

A la suite de ces changements on a diminué la charge fiscale liée à l'acquisition gratuite des objets et droits patrimoniaux, mais cela a d'autre part mené à marginaliser cette source de revenus propres des communes. On estime que les revenus de l'impôt sur l'héritage et les donations venant des acquéreurs du premier groupe d'impôt diminueront a peu près de $65 \%$ à $70 \%$, soit d' environ 165 millions de zlotys par $\mathrm{an}^{23}$.

A partir du $1^{\text {er }}$ janvier 2007, on a renoncé à l'impôt sur les activités de droit civil $^{24}$ du contrat de constitution de rente et des contrats de mariage, des contrats d'emprunt accordés en argent sur la base d'un contrat entre les personnes les plus proches (définies dans l'article 4a de la loi sur l'impôt sur l'héritage et les donations) à condition de déposer la déclaration de revenus dans le délai de 14 jours à partir de la date de l'acte, de prouver la réception de l'argent par l'emprunteur sur son compte bancaire ou sur le compte de celui-ci, tenu par la caisse d'épargne et de crédit, ou par mandat postal, et à condition que le montant de l'emprunt dépasse la somme non soumise à l'impôt, conformément aux principes définis dans les dispositions sur l'impôt sur les héritages et les donations. Si ces conditions ne sont pas satisfaites, l'impôt sur cette activité s'élève à un tarif augmenté, soit à $20 \%$.

On n'a pas fait d'analyse détaillée des effets financiers négatifs de ces changements pour les budgets communaux. On a indiqué en même temps la possibilité d'avoir des recettes budgétaires plus élevées à la suite de: l'imposition des actes de droit civil exempts de la taxe à la valeur ajoutée des contrats de vente et d'échange, dont l'objet est le droit de propriété du local coopératif, le droit à les actes de droit civil.

24 Loi du 9 septembre 2000 sur l'impôt sur les actes de droit civil (Dz. U. z 2007 r. Nr 68, poz. 450 ze zm.). 
une maison dans la société coopérative ou le droit à une place de stationnement dans les garages multipostes ou la participation à ces droits, ainsi que des contrats de vente des parts et actions dans les sociétés de droit commercial; la dérogation à l'exemption de l'impôt des contrats des crédits pour l'activité économique. Tout cela peut contribuer à la hausse des revenus budgétaires d'environ 150 millions de zlotys $^{25}$.

Des changements importants ont été effectués aussi en ce qui concerne l'impôt sur les biens immeubles ${ }^{26}$, entre autres par la hausse ou particularisation du catalogue des exclusions et exemptions de l'impôt. Les terres et bâtiments faisant partie des immeubles destinés à la construction des voies publiques, acquis comme propriété ou gestion durable du Trésor public et transmis à la Direction Générale des Chemins Nationaux et Autoroutes ${ }^{27}$ ont été exempts de l'impôt sur les immeubles. Le catalogue d'exclusions de l'impôt a été élargi des terres sous les eaux maritimes intérieures. Ce changement a pour but d'éliminer les doutes d'interprétation dans le domaine de l'imposition des immeubles des terres sous les eaux maritimes intérieures ${ }^{28}$.

Le contenu de l'article 7 alinéa 1 numéro 1 de la loi sur les impôts et taxes locales a été reformulé, en exemptant de l'impôt les bâtiments faisant partie de l'infrastructure ferroviaire et les terres que celle-ci occupe, et qui satisfont aux conditions formelles définies dans la loi (rendus accessibles aux transporteurs du rail licenciés ou destinés uniquement au transport des personnes, effectué par un transporteur qui a en même temps dans sa gestion cette infrastructure, sans donner l'accès aux autres transporteurs, ou bien créant des lignes de rail dont largeur dépasse $1.435 \mathrm{~mm}$ ). On a en outre exempt de l'impôt les terres, bâtiments et constructions restés après la liquidation des voies ferroviaires ou de leurs segments, jusqu'au moment de cession de leur propriété ou du droit au bail emphytéotique, mais pas plus que pendant 3 ans à partir du premier jour du mois suivant le mois où la décision définitive a été prise ou bien un règlement adéquat est entré en vigueur, approuvant la liquidation des voies ou de leurs segments (la dernière des exemptions mentionnées causera passagèrement un abaissement des recettes budgétaires, et seulement après quelques années peut avoir lieu une augmentation de ces recettes de 70 millions zlotys environ).

25

26

27

De la justification du projet de loi sur le changement de la loi sur les héritages et donations et de l'acte sur l'impôt sur les activités civiles et législatives.

Loi du 12 janvier 1991 sur les impôts et taxes locales (Dz. U. z 2006 r. Nr 121, poz. 844 ze zm.).

Dispositions de la loi du 10 avril 2003 sur les principes de préparation et de réalisation des investissements dans le domaine des voies publiques (loi du 10 avril 2003 sur les principes particuliers de préparation et de réalisation des investissements dans le domaine des voies publiques ; Dz. U. Nr 80, poz. 721 ze zm.) étaient douteux du point de l'interprétation.

Justification du projet de loi sur le changement de la loi sur les impôts et taxes locales et le changement de certaines autres lois - document $n^{\circ} 1082$ de la Diète de la RP de la Vème législature. 
Stabilisation de la régulation des sources de revenus des collectivités territoriales...

Des résultats financiers importants pour les budgets communaux sont causés par les changements dans l'imposition des moyens de transport, en vigueur depuis le $1^{\text {er }}$ janvier 2008. Un changement rédactionnel, apparemment insignifiant, effectué dans l'article 8 de la loi sur les impôts et taxes locales, qui a consisté à remplacer l'expression "à partir de 3,5 tonnes" par "au dessus de 3,5 tonnes" fait qu' on a exclu de l'imposition les camions de masse totale admissible égale à 3,5 tonnes ${ }^{29}$.

Mais les résultats financiers les plus graves sont liés à l'introduction du dégrèvement dans l'impôt sur le revenu des personnes physiques ${ }^{30}$, à titre de la charge des enfants, qui consiste à abaisser le montant de l'impôt sur le revenu calculé de l'équivalent du produit du nombre d'enfants en charge et du double du montant diminuant l'impôt déterminé dans le premier intervalle de 'échelle d'impôt (dans le règlement de l'impôt pour l'année 2007, c'est le montant de 1145,08 zlotys par enfant). On a estimé que la diminution des recettes provenant cet impôt peut s'élever même à 7 milliards de zlotys par an (la part de cet impôt dans les recettes des collectivités locales s'élève, on le sait bien, à presque $50 \%$ ).

\section{Remarques finales}

Du point de vue des intérêts des contribuables, la diminution de la charge de l'impôt est jugée positivement. Mais les contribuables sont aussi membres des communauté locales et bénéficiaires des actions effectuées par celles-ci. L'autorité locale ne peut pas réaliser les objectifs publics d'une façon autonome sans certaines garanties de caractère économique. Des garanties juridiques de cette autonomie résultent des dispositions de l'article 167 de la Constitution de la RP et de l'article 9 de la Charte européenne de l'autonomie locale ${ }^{31}$. Compte tenu de ces standards, il est difficile de juger d'une façon enthousiaste les changements récents des impôts, sources de revenus propres des communes, parce que ceci mène généralement à la diminution des recettes de ces impôts. En plus, la hausse permanente des frais de la réalisation des objectifs dans la collectivité locale est une occurrence négative, causée surtout par la hausse des prix des matériels, d'énergie etc. La diminution de la productivité des sources des revenus propres des communes est généralement compensée dans les collectivités locales par la hausse des prix des services communaux, dont l'accessibilité peut devenir un problème pour des personnes

\footnotetext{
29 La diminution des revenus de budget est estimée à un niveau de 100 à 130 millions de zlotys par an.

30 Loi du 26 juillet 1991 sur l'impôt sur le revenu des personnes physiques (Dz. U. z 2000 r. Nr 14, poz. 176 ze zm.).

31 L. Jędrzejewski, Gospodarka finansowa samorządu terytorialnego w Polsce - wybrane zagadnienia, Oficyna Wydawnicza Branta, Bydgoszcz 2007, p. 35.
} 
moins aisées. Une autonomie limitée des collectivités locales dans le domaine de la formation de leurs revenus ${ }^{32}$ est donc dans les dernières années encore limitée.

Les changements effectués dans la loi sur les revenus des collectivités locales, relatifs aux principes du subventionnement peuvent être un exemple de la "petite stabilisation". En tenant compte de la nécessité de la compatibilité entre la Constitution de la RP et les règlements mis en question par le Tribunal Constitutionnel ${ }^{33}$, on a défini les principes de partager la subvention générale directement dans la loi: dans la partie régionale pour les voïvodies et dans les parties équilibrantes pour les communes et districts. On a renoncé à la régulation annuelle de ces principes dans les arrêtés. Ce changement ne peut être estimé comme entièrement positif parce qu'on s'est limité à introduire dans la loi les arrêtés du Ministre des Finances récemment en vigueur, sans créer des solutions nouvelles et plus claires pour la division des parties de la subvention générale mentionnées. La stabilisation de la situation consiste alors en la fixation législative du partage intérieur en sommes composantes des parties de la subvention générale, auxquelles on applique des critères différents du calcul des montants. 


\section{Streszczenie}

Od momentu przywrócenia samorządu lokalnego w Polsce, tj. od 1990 r., obserwuje się nieustanny proces ulepszania regulacji prawnych i metod finansowania zadań JST. Mimo to system dochodów JST wciąż nie jest stabilny, a kolejne zmiany przynoszą tylko czasową poprawę. Stabilizację należy zatem osiągać, nie tylko $\mathrm{w}$ drodze wydawania aktów prawnych, ale również poprzez unikanie zbyt częstych zmian metod oraz poprzez dostosowanie środków finansowych do wielkosci przekazywanych zadań.

W Polsce standardy te są stopniowo osiągane, a kluczową rolę pełni Trybunał Konstytucyjny, którego decyzje przyczyniają się do zmian ustawodawstwa w zakresie zasad finansowania JST oraz zmian mających na celu dostosowanie regulacji prawnych do postanowień Konstytucji RP i postanowień Europejskiej Karty Samorządu Lokalnego. 